\section{Filarial ascites: A rare cause of non chylous ascites in tropics}

120 million people worldwide are estimated to be affected by filariasis. In the South East and South Asian region, Wuchereria bancrofti is the most prevalent parasite causing lymphatic filariasis (90\% of cases). ${ }^{1}$ Microfilaria has already been found in many specimens like pleural fluid and also in breast, pancreas and thyroid tissue. ${ }^{2}$ Ascites resulting because of filariasis is low serum ascites albumin gradient (SAAG) ascites, typically chylous in nature due to lymphatic obstruction.

\section{Case Report}

This 28 year nonalcoholic male patient presented with insidious onset gradually increasing abdominal distension since 4 months. There was no history of gastrointestinal bleed, decrease urine output, jaundice, intestinal obstruction, fever. Physical examination showed tense ascites, but there were neither signs of the liver cell failure nor hepatosplenomegaly. Complete blood count revealed, $\mathrm{Hb} \%=12.3 \mathrm{gm} / \mathrm{dl}, \mathrm{TLC}=11,480 / \mathrm{mm}^{3}, \mathrm{~N}=75.7 \%$, $\mathrm{L}=13.5 \%, \mathrm{E}=1.7 \%$. Liver and kidney functions are were within normal limits. On diagnostic paracentesis, ascitic fluid (AF) was straw coloured. AF analysis revealed low SAAG of 0.6. ADA was 20.6 U/L, triglycerides were $5 \mathrm{mg} / \mathrm{dl}$. H\& E and May Grunwald Giemsa (MGG) stained smears showed plenty of reactive mesothelial cells, lymphocytes and RBCs in the background. Sheathed Microfilariae were seen in the ascitic fluid. Cytological preparations suggestive of $W$. Brancrofti (Figure 1A,B). No malignant cells were seen. Midnight peripheral blood smear showed microfilaria of the $W$. bancrofti (Figure 1C). Abdominal ultrasound showed gross ascites and scrotal ultrasound was done in view of finding microfilaria in blood which showed echogenic, undulating structure with persistent twirling motion suggestive of filarial worms (Figure 1D "filarial dance" sign). Workup for the ascites including ESR, ANA, $24 \mathrm{hr}$ urinary copper, serum ceruloplasmin, $\mathrm{KF}$ ring, $\mathrm{HBsAg}$, HCV, 2D ECHO, Lipid Profile, ascitic fluid amylase were normal. Upper gastrointestinal endoscopy showed no varices. Considering the diagnosis of filarial ascites patient was started with antifilarial treatment. Single dose of ivermectin followed one month course of diethylcarbamazine with which patient's ascites was completely resolved on follow up at one month and ascites has not refilled after three months of follow up.

\section{Discussion}

Infection with three closely related filarial wormsWuchereria bancrofti, Brugia malayi and Brugia timori causes lymphatic filariasis. Bancroftian filariasis is caused by $W$. bancrofti. In about $90 \%$ cases of lymphatic filariasis is due to $W$. bancrofti and for the remaining $10 \%$ cases Brugia malayi and Brugia timoriare are responsible. ${ }^{1}$ Bancroftian filariasis produce wide spectrum of clinical manifestations. The acute phase is characterized by fever, lymphangitis, lymphadenitis, epididymo-orchitis, and funniculitis. Chronic stage of Bancroftian filariasis is characterized by lymphadenopathy, lymphedema, hydrocele, and elephantiasis. A significant number of infected individuals in endemic areas remain asymptomatic

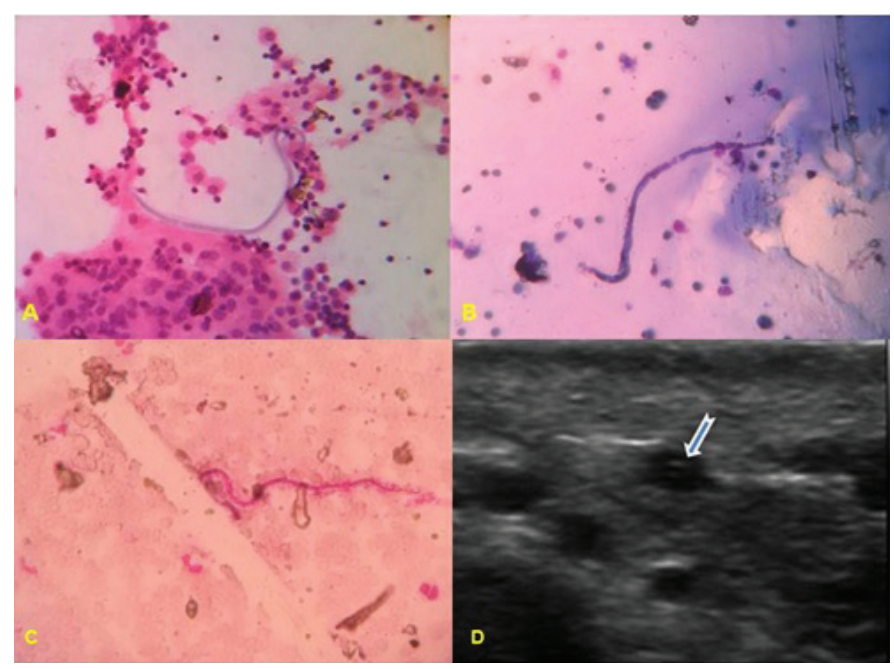

Figure 1: A (H \& E stain 10X): Ascitic fluid Cytological smear showing a single microfilaria of Wuchereria bancrofti along with a cluster of reactive looking mesothelial cells. (B): MayGrunwald-GiemsaStain (MGG) 10X showing microfilaria in ascitic fluid. (C): Peripheral blood smear showing the microfilaria in $\mathrm{H} \& \mathrm{E}$ staining. (D): Ultrasound of Scrotum showing The Filarial Worm. 
throughout their life. ${ }^{2}$ The chronic and most debilitating sequelae of lymphatic filariasis often develop years after initial infection. ${ }^{3}$ Chylous ascites is characterized by chyle in the peritoneal cavities produced by obstruction and disruption of the retroperitoneal lymphatic channel. ${ }^{4}$ The diagnosis of microfilaria is conventionally made by demonstration of microfilaria in the peripheral blood smear. Pathogenesis of nonchylous filarial ascites remains enigmatic. Lymphangitis because of partial obstruction is a postulated cause for such nonchylous low SAAG (exudative) ascites. ${ }^{5}$

NITIN R. GAIKWAD

SUDHIR J. GUPTA

AMOL R. SAMARTH

TUSHAR H. SANKALECHA

Department of Gastroenterology, Government Medical College and Super Speciality Hospital, Nagpur, Maharashtra,

India.

Correspondence: Sudhir Gupta

Email:sudhirjgupta@gmail.com

\section{References}

1. Mannan R, Bhasin TS, Manjari M, Misra V. Low density microfilaraemia as a causative agent in a case of unexplained ascites of suspicious nature. J Clin Diagn Res. 2013;7(7):1441-3.

2. Meyers WM, Neafi RC, Connor DH. Edited by Binford C.H., Connor D.H. Diseases caused by filarial NematodesBancroftian and Malayan Filariasis Pathology of Tropical and Extraordinary diseases. An Atlas.Vol II. Washington D.C: Armed Forces Institute of Pathology. 1976.p.340-55.

3. Partono F. The spectrum of disease in lymphatic filariasis. Ciba Found Symp. 1987;127:15-31.

4. Aalami OO, Allen DB, Organ CH Jr. Chylous ascites: a collective review. Surgery. 2000;128:761-7.

5. Walter A, Krishnaswami H, Cariappa A. Microfilariae of Wuchereria bancrofti in cytologic smears. Acta Cytol. 1983;27:432-436. 